\title{
A Practical Method of Nonprobabilistic Reliability and Parameter Sensitivity Analysis Based on Space-Filling Design
}

\author{
Xin-dang He, Wen-xuan Gou, Yong-shou Liu, and Zong-zhan Gao \\ Department of Engineering Mechanics, Institute of Aircraft Reliability Engineering, Northwestern Polytechnical University, \\ Xian 710129, China \\ Correspondence should be addressed to Xin-dang He; xindanghe@163.com
}

Received 21 July 2014; Revised 29 October 2014; Accepted 10 November 2014

Academic Editor: Shaofan Li

Copyright (C) 2015 Xin-dang He et al. This is an open access article distributed under the Creative Commons Attribution License, which permits unrestricted use, distribution, and reproduction in any medium, provided the original work is properly cited.

Using the convex model approach, the bounds of uncertain variables are only required rather than the precise probability distributions, based on which it can be made possible to conduct the reliability analysis for many complex engineering problems with limited information. In this paper, three types of convex model including interval, ellipsoid, and multiellipsoid convex uncertainty model are investigated, and a uniform model of nonprobabilistic reliability analysis is built. In the reliability analysis process, an effective space-filling design is introduced to generate representative samples of uncertainty space so as to reduce the computational cost and provide an accurate depiction of possible model outcome. Finally, Spearman's rank correlation coefficient is used to perform parameters global sensitivity analysis. Three numerical examples are investigated to demonstrate the feasibility and accuracy of the presented method.

\section{Introduction}

In practical engineering problems, uncertainty is often unavoidable due to the lack of knowledge, assumptions made by model builders, variations of physical properties of materials, geometric dimensions, and operating environments and other reasons. Therefore, these uncertainties involved in structure should be taken into account for a proper design process [1]. With the ever-increasing demands of structure security, the structural reliability analysis has received considerable attention in the last decades and is becoming increasingly important in the structural design [2].

The aim of reliability analysis is to assess the probability of structural survival or the probability of structural failure when the uncertainties involved in the structures are take into account [3]. In the area of reliability analysis, probability model is one of the most commonly used uncertainty models, in which the uncertainties involved in structures are described as random variables. This reliability model has been intensively studied in the last decades and a variety of important analysis techniques have been established, such as the first order reliability method (FORM) $[4,5]$, second order reliability method (SORM) [6,7], Monte-Carlo method (MC) $[8]$, and system reliability method $[9,10]$.

It is important to point out that the aforementioned reliability method requires precise probabilistic distribution characteristics, which come from a large number of input samples. However, in real world engineering design problems, probabilistic reliability model is often hindered due to the lack of samples information. For instance, in the conceptual design stage of structures, it is difficult to provide sufficient experimental samples to describe the distribution of uncertainty input variables [11]. As revealed by Ben-Haim [12] and Elishakoff [13], probabilistic reliability model is very sensitive to small inaccuracy of input quantities, which means that misleading results may be yield due to the unjustified assumption in constructing a probabilistic model.

This challenge thus raised the interest of the alternative nonprobabilistic approach for uncertainty description, namely, the set theory-based convex model [14-18]. At present, interval, ellipsoid, and multiellipsoid convex model are three widely studied convex models [19]. Based on interval model, Guo et al. [20] proposed a robust reliability index to estimate structural nonprobability reliability and 
presented three solution methods of the reliability index. Tao et al. [21] proposed a semianalytic method to solve the nonprobabilistic reliability index. Chen et al. [22] suggested an enhanced semianalytic method based on monotonicity analysis. Qiu and Elishakoff studied the structural optimal design method using the interval set model [23, 24]. With multiellipsoid convex model, Kang et al. [25] and Luo et al. [26] constructed a nonprobabilistic reliability optimization design method.

This paper aims to develop a practical nonprobability reliability global sensitivity analysis technique based on optimal Latin hypercube sampling (OLHS) and Monte Carlo simulation. The method proposed in this paper has wider applicability. It can be used in solving more general problems with interval model, ellipsoid model, or multiellipsoid convex model. The proposed method has some very attractive advantages, such as the following: its analysis process is simple, computational cost is regardless with variables dimension and the nonlinear degree of the performance function. Hence it is particularly suitable for implementing the nonprobabilistic reliability analysis for some complicated structure.

This paper is organized as follows. Firstly, three uncertain information description methods, including interval model, ellipsoid model, and multiellipsoid convex model, are introduced. The nonprobabilistic reliability model and its physical meaning are then discussed in detail in Section 3. Based on OLHS, a Monte Carlo simulation and global sensitivity analysis method for structural nonprobabilistic reliability is proposed in Section 4. The feasibility and accuracy of the presented method are demonstrated through three numerical examples in Section 5. Conclusions are given in Section 6.

\section{The Description of Structural Uncertainty with Convex Model}

In this paper, three types of ellipsoid-bound convex models, including interval model, ellipsoid model, and multiellipsoid convex model, are introduced for the description of uncertainty variables $[14,27]$.

(1) In interval uncertainty model, an uncertain variable can be described by an interval set, which can be expressed as

$$
x=\left[x^{L}, x^{U}\right]=\left\{x \in R \mid x^{L} \leq x \leq x^{U}\right\},
$$

where variable $x \in R$ belongs to a bounded closed interval, $x^{L}$ denotes the lower bound of interval variable, $x^{U}$ stands for the upper bound of interval variable, and $x^{L} \leq x^{U}$. by

For easy expression, the variable $x$ can also be expressed

$$
x=(1+\delta) \cdot \bar{x}
$$

where $\delta$ denotes a dimensionless variable and $\bar{x}$ denotes the nominal value of the uncertain variable; it can be expressed by

$$
\bar{x}=\frac{x^{L}+x^{U}}{2} \text {. }
$$

(2) In ellipsoid uncertain model, the uncertain variables are assumed to fall into a multidimensional (hyper-) ellipsoid. The uncertainty domain can be expressed by

$$
\boldsymbol{\delta}=\left\{\boldsymbol{\delta}: \boldsymbol{\delta}^{T} \mathbf{W} \boldsymbol{\delta} \leq \varepsilon^{2}\right\}
$$

where $\mathbf{W}$ is the characteristic matrix of the ellipsoid and $\varepsilon$ is real number standing for the magnitude of the parameter variability.

(3) In multiellipsoid uncertain model, the uncertain variables are assumed to fall into several ellipsoid sets. Supposing $k$ ellipsoid sets are employed, the vector of the grouped uncertain variables can be expressed by

$$
\mathbf{x}^{T}=\left\{\mathbf{x}_{1}^{T}, \mathbf{x}_{2}^{T}, \ldots, \mathbf{x}_{k}^{T}\right\}
$$

where each group of variables is bounded by an ellipsoid set as follows:

$$
\boldsymbol{\delta}_{i}=\left\{\boldsymbol{\delta}_{i}: \boldsymbol{\delta}_{i}^{T} \mathbf{W}_{i} \boldsymbol{\delta}_{i} \leq \varepsilon_{i}^{2}\right\} \quad(i=1,2, \ldots, k),
$$

where $\boldsymbol{\delta}_{i}$ denotes the dimensionless vector of $\mathbf{x}_{i}, \mathbf{W}_{i}$ denotes the characteristic matrix of the $i$ th ellipsoid, and $\varepsilon_{i}(i=$ $1,2, \ldots, k)$ are real numbers.

For illustrative purpose, we provide a problem with three uncertain variables. Its dimensionless vector is expressed by $\boldsymbol{\delta}=\left\{\delta_{1}, \delta_{2}, \delta_{3}\right\}^{T}$. We describe it with interval model, ellipsoid model, and multiellipsoid convex model, respectively.

(a) Interval model:

$$
\delta_{1}^{2} \leq \varepsilon_{1}^{2}, \quad \delta_{2}^{2} \leq \varepsilon_{2}^{2}, \quad \delta_{3}^{2} \leq \varepsilon_{3}^{2} .
$$

(b) Single-ellipsoid model:

$$
\left\{\delta_{1}, \delta_{2}, \delta_{3}\right\}\left[\begin{array}{lll}
3 & 0 & 1 \\
0 & 3 & 1 \\
1 & 1 & 1
\end{array}\right]\left\{\begin{array}{l}
\delta_{1} \\
\delta_{2} \\
\delta_{3}
\end{array}\right\} \leq \varepsilon^{2} .
$$

(c) Multiellipsoid uncertain model:

$$
\left\{\delta_{1}, \delta_{2}\right\}\left[\begin{array}{ll}
1 & 0 \\
0 & 1
\end{array}\right]\left\{\begin{array}{l}
\delta_{1} \\
\delta_{2}
\end{array}\right\} \leq \varepsilon_{1}^{2}, \quad \delta_{3}^{2} \leq \varepsilon_{2}^{2} .
$$

The above three types of convex model are schematically shown in Figures 1(a)-1(c).

\section{A Reliability Model with Convex Uncertainty}

During the reference review, we found that Jiang et al. [28] proposed a desirable structural nonprobabilistic reliability model, in which reliability is defined as a ratio of the multidimensional volume falling into the reliability domain to the one of whole convex model. This reliability model has a very intuitional and easily understandable mathematical form and can be used to deal with some complex engineering problems. In this reference, ellipsoid convex model is intensively discussed, while, in this paper, this reliability model 


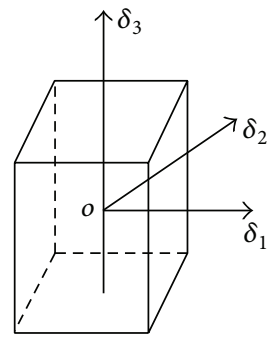

(a) Interval model

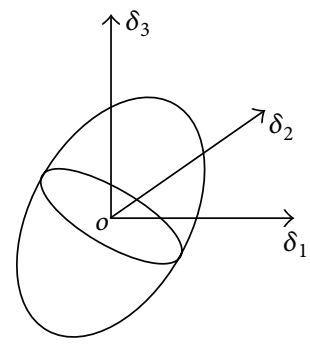

(b) Single-ellipsoid model

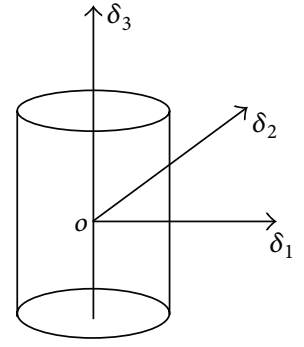

(c) Multiellipsoid model

FIGURE 1: Convex models for three uncertain variables.

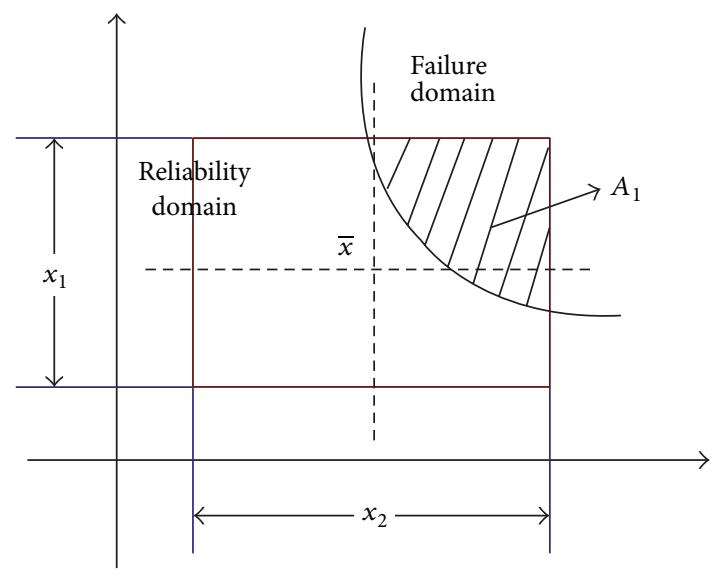

(a) Interval model

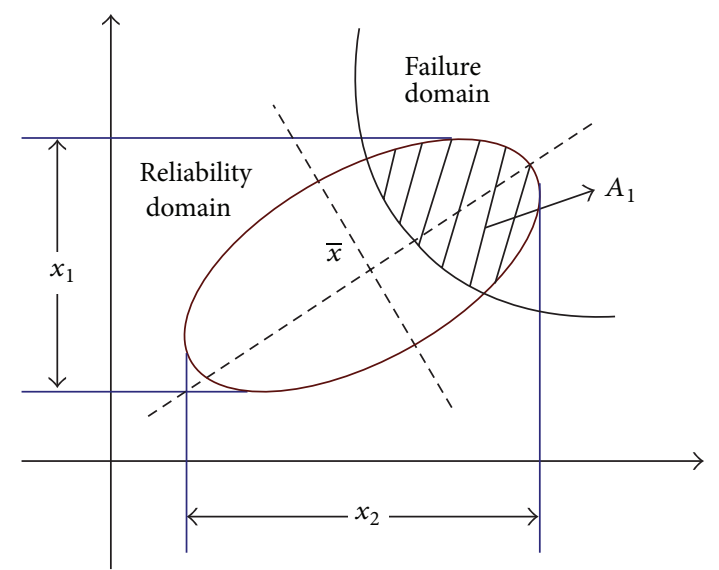

(b) Single-ellipsoid model

FIgURE 2: Nonprobabilistic reliability for 2D problem.

will be expanded to interval model, ellipsoid model, and multiellipsoid convex model, which will be able to deal with more general convex model problems. Assume a structural performance function is expressed by

$$
Y=g(\mathbf{x}),
$$

where $\mathbf{x}$ stands for an $n$-dimensional vector of input parameters.

Firstly, a two-dimensional problem is considered. As shown in Figure 2

From Figure 2, the whole uncertainty domain is divided into two regions by the failure surface. One is in the reliability region, which is denoted by $g(\mathbf{x}) \geq 0$. The other one is in the failure region, which is denoted by $g(\mathbf{x})<0$. The reliability can be evaluated as follows:

$$
R_{c}=\frac{A-A_{1}}{A},
$$

where $A$ stands for the region of the whole convex model, $A_{1}$ stands for the failure region, and $A-A_{1}$ stands for the reliability region.

When a three-dimensional problem is taken into account, the domain of whole convex model will extend to corresponding volumes as shown in Figure 3.

The definition of the nonprobabilistic reliability formulated by (11) can easily be extended to an $n$-dimensional problem, in which the domain will become a multidimensional volume. The value of $R_{c}$ is in the range of $[0,1]$, which can well describe the safety extent of a structure. Correspondingly, the dangerous degree $f_{c}$ can be expressed by

$$
f_{c}=\frac{A_{1}}{A} .
$$

Obviously, we can find that the relationship of dangerous degree $f_{c}$ with reliability $R_{c}$ can be expressed by

$$
R_{c}+f_{c}=1
$$

\section{Reliability and Variables Global Sensitivity Solution Method Based on Optimal Latin Hypercube Sampling}

It should be pointed out that the reliability model mentioned above is based on areas or volumes. However, the magnitude of reliability domain in the whole uncertainty domain is difficult to be computed analytically for some complex engineering problems. Thus, a Monte Carlo simulation method has been developed in [28] to deal with this problem. However, the Monte Carlo simulation present in this reference can not assure the samples are uniformly scattered in the uncertainty space. Hence the number of samples falling into reliability domain can not effectively represent the magnitude 


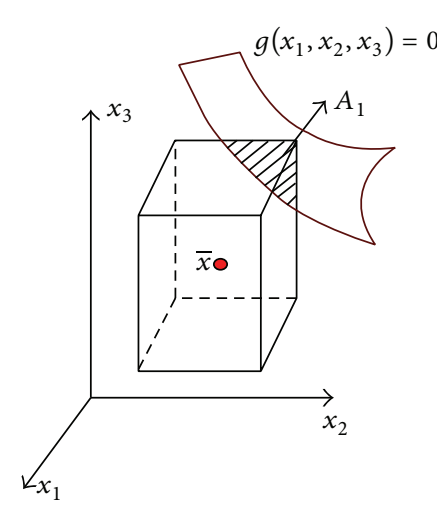

(a) Interval model

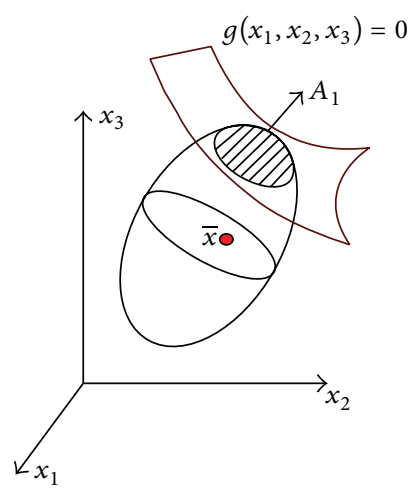

(b) Single-ellipsoid model

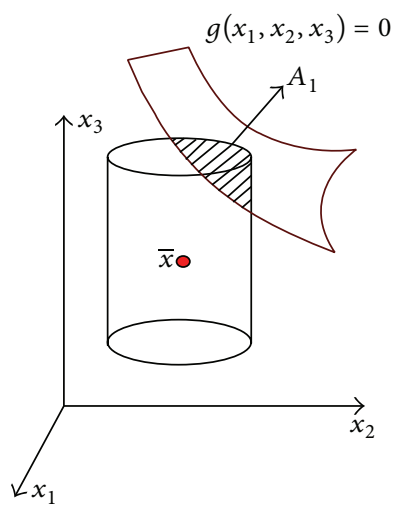

(c) Multiellipsoid model

Figure 3: Nonprobabilistic reliability for 3D problem.

of reliability domain. The precision of analysis result can not be ensured even though a lot of samples are used.

In this study, we approached the problem by a spacefilling design of computer experiment. This method is proposed by Jin et al. [29] and named as optimal Latin hypercube sampling (OLHS). Because OLHS can evenly spread out sample points over the entire design space, people also named it as space-filling design [30]. After samples of structural uncertainty variables are generated readily by the OLHS in Section 4.1, an efficient nonprobabilistic reliability and global sensitivity analysis method will be proposed in Sections 4.2 and 4.3 , respectively.

4.1. Generate the Samples of Convex Model Uncertainty Variables Based on Uniform Design. When the uncertain variables are defined as (tolerances) intervals with given lower and upper bounds, failure analysis methods are needed to consider the complete range of uncertain domain. Theoretically, every point within the interval has some unknown probability of occurrence. Therefore, all the possible combinations of these uncertain intervals must be considered in the analysis.

In this section, we will use optimal Latin hypercube sampling (OLHS) to solve structural nonprobabilistic reliability index. OLHS is one of the space-filling designs and it seeks experimental points to be uniformly scattered in the experimental domain, which is proposed by Jin et al. [29, 30].

OLHS has several advantages. It can explore relationships between the response and the factors with a reasonable number of runs and is shown to be robust to the situation where little knowledge is known about the function to be modeled. In the past decade, it has been successfully applied in industry, system engineering, pharmaceutics, and natural sciences. Its practical success is due to its economical and flexible experimental runs to study many factors with high levels simultaneously.

In order to verify the space-filling capability of OLHS, a graphical comparison of Monte Carlo sampling (MCS), Halton sequence, Latin square sampling (LHS), Maximin Latin hypercube sampling (Maximin LHS), and Optimal Latin hypercube (Optimal LHS) for two independent variables is presented in Figure 4.

As shown in Figure 4, among these sampling methods, the OLHS method has been shown to be capable of spacefilling uniformity. It can uniformly scatter samples in the 2D space and obviously is better than the other methods in terms of space-filling capability. Hence, in this paper, OLHS will be introduced to solve the nonprobabilistic index. Concerning how to carry out the optimal Latin hypercube sampling, this content is discussed detailedly in [25]. So we will not repeat it in this paper.

Consider that a structure contains $S$ variables that can be realized $N$ times. The samples of input variables can be arranged as an input matrix with $N$ row and $S$ columns. Using OLHS to generate $N \times S$ uniform numbers $r_{i}$ in [0 1], arrange these values into a $N \times S$ matrix, which has the smallest discrepancy and excellent space-filling capability. After the matrix $N \times S$ in $[0,1]$ has been generated, we will construct the sampling matrix $N \times S$ for three types of uncertainty convex model including interval model, ellipsoid model, and multiellipsoid convex model.

(1) Interval Model. For interval model, uncertain variables can be described by an interval set with upper and lower bounds (Figure 5). The uncertain domain can be expressed by

$$
\Omega_{1}=\left\{x=\left(x_{i}, \ldots, x_{S}\right): x_{i}^{L} \leq x_{i} \leq x_{i}^{U}, i=1, \ldots, S\right\},
$$

where variable $x_{i} \in R$ and belong to a bounded closed interval, $x_{i}^{L}$ is the lower bound of interval variable, $x_{i}^{U}$ is the upper bound of interval variable, and $x_{i}^{L} \leq x_{i}^{U}$.

Consider a computer model requiring $S$ variables that can be realized $N$ times. After a matrix $N \times S$ in $[0,1]$ has been generated, we will construct the sampling matrix by

$$
x_{i}^{*}=x_{i}^{L}-r_{i}^{*}\left(x_{i}^{U}-x_{i}^{L}\right),
$$

where $x_{i}^{*}$ is the sample of interval variable and $r_{i}^{*}$ is the sample of matrix $N \times S$ in $[0,1]$.

(2) Ellipsoid Model. For ellipsoid convex model, it is difficult to obtain the samples directly. Hence we can make the $n$ 

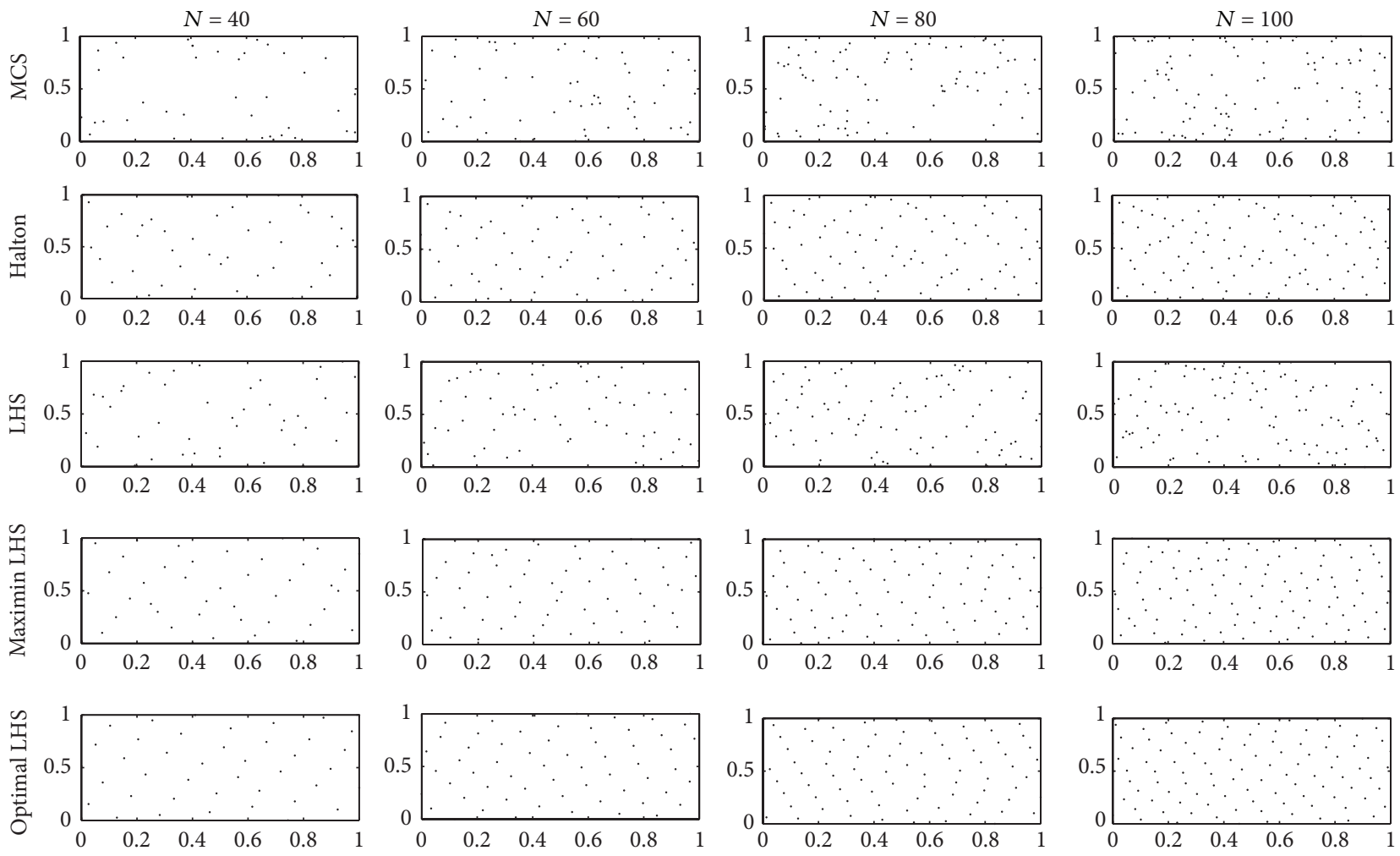

FIgURE 4: Comparison of realization sets generated by MCS, Halton, LHS, Maximin LHS, and OLHS.

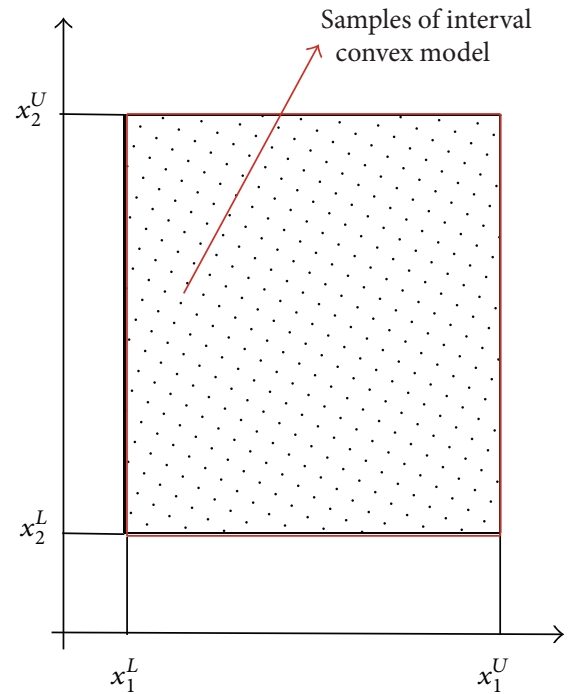

Figure 5: Samples of interval model variables in 2D space.

uncertain parameters as independent random variables and obtain samples by interval model at first. Then we substitute the samples into the ellipsoidal function and obtain a pile of samples $\mathbf{X}_{\mathrm{e}}^{*}$ satisfying

$$
\Omega_{2}=\left\{\mathbf{X}_{\mathrm{e}}^{*}: \boldsymbol{\delta}^{T} \mathbf{W} \boldsymbol{\delta} \leq \varepsilon^{2}\right\} .
$$

By this treatment, we can get a pile of samples, which can be uniformly scattered in the $n$-dimensional ellipsoidal convex model. This principle is shown in Figure 6.

(3) Multiellipsoid Model. Similarly to ellipsoid convex model, we can make the $n$ uncertain parameters as independent random variables and obtain samples by interval model at first. Then we substitute the samples into the multiellipsoidal function and obtain a pile of samples $\mathbf{X}_{\mathrm{me}}^{*}$ satisfying

$$
\Omega_{3}=\left\{\mathbf{X}_{\mathrm{me}}^{*}: \boldsymbol{\delta}_{i}^{T} \mathbf{W}_{i} \boldsymbol{\delta}_{i} \leq \varepsilon_{i}^{2}, i=1,2, \ldots, k\right\} .
$$

By this treatment, we can get a pile of samples $\mathbf{X}_{\mathrm{me}}^{*}$, which can be uniformly scattered in the $n$-dimensional multiellipsoidal convex model.

4.2. Reliability Solution Method of Convex Models Based on Monte Carlo Simulation. In the process of reliability solution, we calculate the number of samples instead of the magnitude of failure region. The proposed reliability method can be outlined as follows.

Step 1. Consider a computer model containing $S$ variable; we first generate the input variables samples matrix by the method mentioned in Section 4.1. Suppose the number of generated samples is $N$; the samples of input variables can be viewed as an input matrix with $N$ row and $S$ columns, 


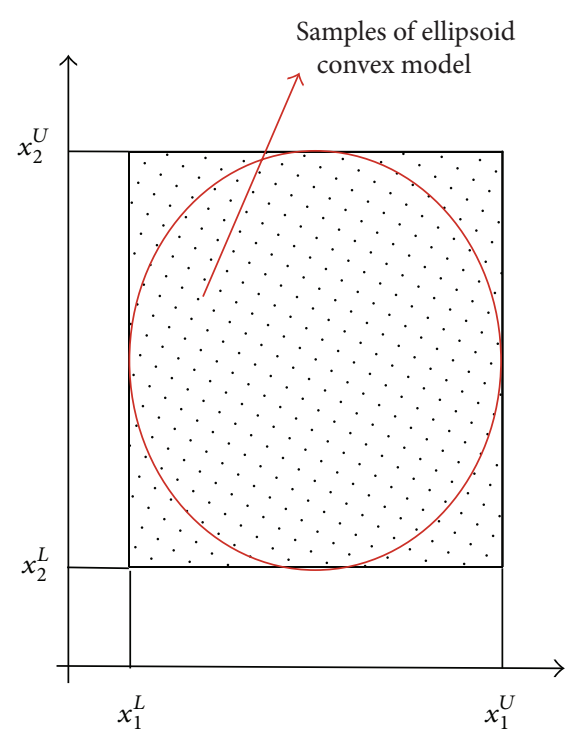

FIGURE 6: Samples of ellipsoid convex model variables in 2D space.

which has the smallest discrepancy and excellent space-filling capability.

Step 2. Sequentially substitute the samples into the performance function. Then we can obtain the number of samples satisfying the $g(\mathbf{x})=g\left(x_{1}, x_{2}, \ldots, x_{n}\right)>0$. We denoted it by $N_{s}$. Through this step, the samples falling into the reliability domain can be captured.

Step 3. Finally, calculate structural reliability through the equation $R_{c}=N_{s} / N$.

4.3. Global Sensitivity Analysis Method of Convex Uncertainty Model. Compared with the researches on solving nonprobabilistic reliability index, the nonprobabilistic reliability sensitivity analysis is less available. Sensitivity analysis can quantitatively assess the impact of inputs on the output, which can be used to identify significant inputs and thereby to help you to improve design toward a more reliable and better quality product.

In this paper, we will present a global sensitivities analysis approach, different from local sensitivities [31]. Local sensitivities are mostly only local gradient information. It usually calculates structural variables sensitivity by finite-differencing scheme. Local sensitivities cannot globally reflect the impact of the variability of inputs on the output. It also neglects the influence of interactions between inputs [32]. The global approach proposed in this paper cannot only consider the slope at a particular location, but also study the global variability of an output induced by inputs over the entire range of values of inputs. Hence it can provide an overall view on the influence of inputs on the outputs.

In this paper, OLHS and Spearman's rank correlation coefficient [33] will be introduced to quantitatively assess the influence of the inputs on the output performance function.
Suppose the samples of input variables are denoted by $x_{1}, x_{2}, \ldots, x_{n}$, and simulation results of output are denoted by $y_{1}, y_{2}, \ldots, y_{n}$. Then we can calculate Spearman's rank correlation coefficient $r_{s}$ as follows:

$$
r_{s}=\frac{\sum_{i=1}^{n}\left(R_{x_{i}}-\bar{R}_{x}\right)\left(R_{y_{i}}-\bar{R}_{y}\right)}{\sqrt{\sum_{i=1}^{n}\left(R_{x_{i}}-\bar{R}_{x}\right)^{2}} \sqrt{\sum_{i=1}^{n}\left(R_{y_{i}}-\bar{R}_{y}\right)^{2}}},
$$

where $R_{x_{i}}$ is the rank of $x_{i}$ within the set of samples $x_{1}, x_{2}, \ldots, x_{n} . R_{y_{i}}$ is the rank of $y_{i}$ within the set of samples $y_{1}, y_{2}, \ldots, y_{n} \cdot \bar{R}_{x}$ denotes the average ranks of $R_{x_{i}}$, and it can be calculated by $\bar{R}_{x}=\sum_{i=1}^{n} R_{x_{i}} / n=(n+1) / 2 . \bar{R}_{y}$ denotes the average ranks of $R_{y_{i}}$, and it can be calculated by $\bar{R}_{y}=$ $\sum_{i=1}^{n} R_{y_{i}} / n=(n+1) / 2$. Equation (18) can be rewritten in a simple manner as follows:

$$
r_{s}=1-\frac{6\left[\sum_{i=1}^{n}\left(R_{y_{i}}-R_{x_{i}}\right)^{2}\right]^{2}}{n\left(n^{2}-1\right)}, \quad-1 \leq r_{s} \leq 1 .
$$

The range of the value $r_{s}$ is in the range of -1 to 1 . Its magnitude stands for the extent of closeness between the input variable and output. A positive value will be obtained if the variables are directly positively related, while a negative value will be obtained if they are inversely related.

Consider a computer model containing $S$ variables. We generate $N$ samples by uniform design. Then the Monte Carlo simulation will be performed to obtain the sampling results of performance function, which can be denoted by $\left\{y_{1}, y_{2}, \ldots, y_{N}\right\}$. We can organize the input parameter and output parameter as follows:

$$
\left[\begin{array}{cccc}
x_{11} & x_{12} & \ldots & x_{1 S} \\
x_{21} & x_{22} & \ldots & x_{2 S} \\
\vdots & \vdots & \vdots & \vdots \\
x_{N 1} & x_{N 2} & \ldots & x_{N S}
\end{array}\right]\left[\begin{array}{c}
y_{1} \\
y_{2} \\
\vdots \\
y_{N}
\end{array}\right]
$$

By the definition of Spearman's rank correlation coefficient, we can divide the input parameter and output parameter into $S$ groups as follows:

$$
\left[\begin{array}{c}
x_{11} \\
x_{21} \\
\vdots \\
x_{N 1}
\end{array}\right]\left[\begin{array}{c}
y_{1} \\
y_{2} \\
\vdots \\
y_{N}
\end{array}\right],\left[\begin{array}{c}
x_{12} \\
x_{22} \\
\vdots \\
x_{N 2}
\end{array}\right]\left[\begin{array}{c}
y_{1} \\
y_{2} \\
\vdots \\
y_{N}
\end{array}\right], \ldots,\left[\begin{array}{c}
x_{1 S} \\
x_{2 S} \\
\vdots \\
x_{N S}
\end{array}\right]\left[\begin{array}{c}
y_{1} \\
y_{2} \\
\vdots \\
y_{N}
\end{array}\right]
$$

By performing nonparametric Spearman's rank correlation coefficient analysis, we can obtain the sensitivity information of variables about structural performance function. The advance of this method is that it does not increase the computation cost. After the reliability analysis is implemented, the sensitivity results can be obtained readily.

\section{Engineering Examples and Discussion}

5.1. Case Study 1. In order to expound the analysis process of the proposed method easily, a simple finite element analysis (FEA) model is investigated. 
TABLE 1: Uncertainty variables of plate structure.

\begin{tabular}{lccc}
\hline Variable name & Mean value & Minimum & Maximum \\
\hline Length $A(\mathrm{~mm})$ & 95 & 93 & 97 \\
Width $B(\mathrm{~mm})$ & 40 & 38 & 42 \\
Height $H(\mathrm{~mm})$ & 6 & 5 & 7 \\
Young's modulus $E(\mathrm{MPa})$ & 71700 & 71600 & 71800 \\
External load $F(\mathrm{~N})$ & 2000 & 1900 & 2100 \\
\hline
\end{tabular}

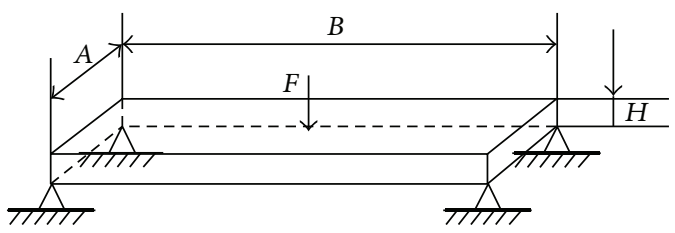

Figure 7: A plate structure.

This model is a simple plate structure as shown in Figure 7. Its length, width, and height are denoted by $A, B$, and $H$, respectively. In this model, an external load denoted by $F$ is acted at the center of the upper surface, and four corners of the lower surface are fixed. Young's modulus of the plank is denoted by $E$, and Poisson's ratio is 0.33 . The uncertainties related to geometrical dimensions, material properties, and external loads can be described with interval model and are listed in Table 1.

The FEA model of the plate structure is initially built with the mean value of variables, and structural response (maximum strain) is shown in Figure 8.

Suppose the maximum strain of the plate is denoted by $d(A, B, H, E, F)$ and the allowable strain of the plane is $d_{m}=$ $0.5 \mathrm{~mm}$; we can define structural performance function as follows:

$$
g(A, B, H, E, F)=d_{m}-d(A, B, H, E, F) .
$$

To perform Monte Carlo analysis with 1000 samples generated by uniform design, the plane structural reliability can be readily obtained by $R_{c}=(1000-9) / 1000=0.991$. The sampling results of performance function are shown in Figure 9.

To implement the sensitivity analysis by the method mentioned in Section 4.3, the bar chart of sensitivity results is shown in Figure 10.

As shown in Figure 10, the variable $H$ is a main factor that influences the magnitude of structural strain. Compared with other variables, Young's modulus $E$ has less impact on the magnitude of structural strain.

5.2. Case Study 2. A practical 25-bar steel truss (Figure 11) is investigated, which is modified from [28].

In this model, Young's modulus is $199949.2 \mathrm{MPa}$ and Poisson's ratio is 0.3 . Horizontal bars and the vertical bars have the same length denoted by $L$. The cross-sectional area of bars (1)-(4) is $A_{1}$, the cross-sectional area of bars (16)-(25) is $A_{2}$, the cross-sectional area of bars (11)-(15) is $A_{3}$, and the cross-sectional area of bars (5)-(10) is $A_{4}$. The joints 6,8 , and

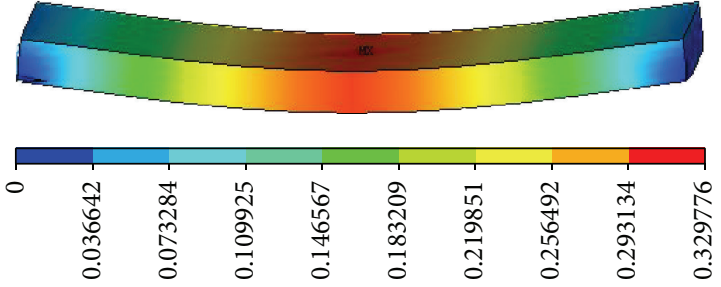

FIgURE 8: Strain of the plate structure.

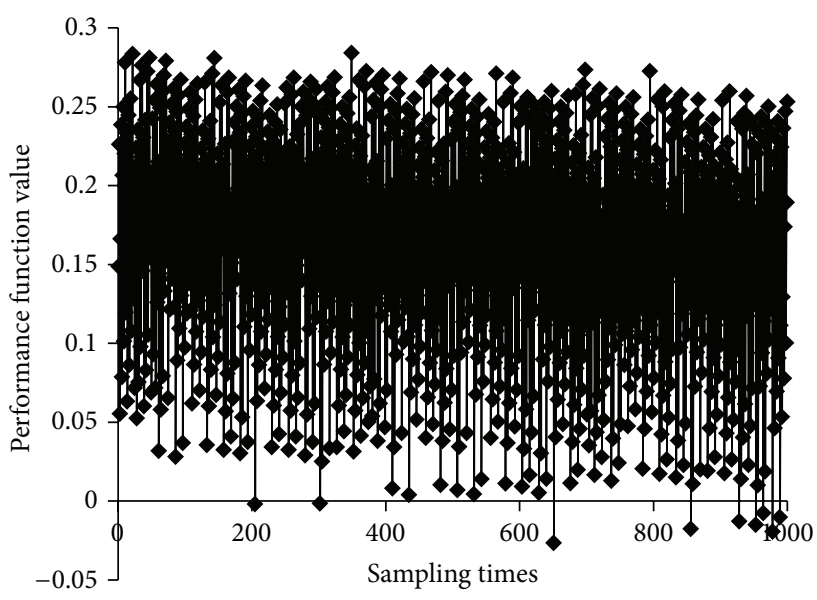

FIGURE 9: Sampling results of performance function.

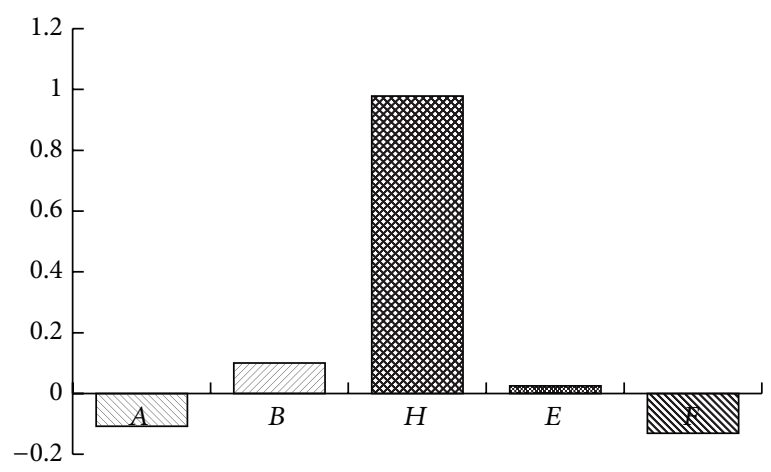

FIGURE 10: Bar chart of sensitivity analysis results.

10 are roller-supported, and joint 12 is hinge-supported. The vertical loads are denoted by $F_{3}, F_{2}$, and $F_{1}$ and are acted at the joints 7,9 , and 10, respectively. A horizontal load is denoted by $F_{4}$ and is acted at the joint 1 . The horizontal displacement of joint 6 is denoted by $d$, and its allowable maximum value is $d_{m}$. The five inputs (cross-sectional area $A_{i}, i=1,2,3,4$, and the length $L$ ) in this example are treated as uncertain variables. The performance function of the structure can then be expressed as

$$
g\left(A_{1}, A_{2}, A_{3}, A_{4}, L\right)=d_{m}-d\left(A_{1}, A_{2}, A_{3}, A_{4}, L\right) .
$$




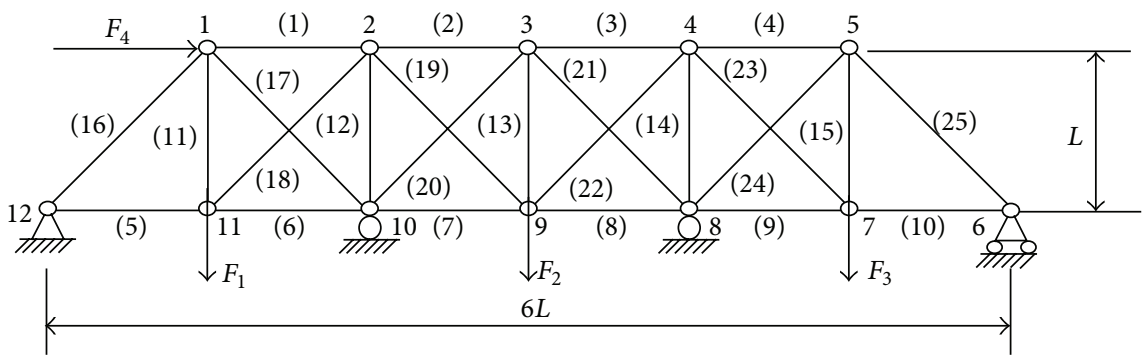

FIGURE 11: A 25-bar truss.

The uncertain intervals of the five input variables are

$$
\begin{aligned}
A_{1} & =\left[630 \mathrm{~mm}^{2} ; 770 \mathrm{~mm}^{2}\right], \\
A_{2} & =\left[5580 \mathrm{~mm}^{2}, 6820 \mathrm{~mm}^{2}\right], \\
A_{3} & =\left[4770 \mathrm{~mm}^{2} ; 5830 \mathrm{~mm}^{2}\right], \\
A_{4} & =\left[7920 \mathrm{~mm}^{2} ; 9680 \mathrm{~mm}^{2}\right], \\
L & =\left[13500 \mathrm{~mm} ; 16500 \mathrm{~mm}^{2}\right] .
\end{aligned}
$$

The uncertainty domain of the design problem can be represented as an ellipsoidal convex model:

$$
\begin{aligned}
& {\left[\begin{array}{c}
A_{1}-\bar{A}_{1} \\
A_{2}-\bar{A}_{2} \\
A_{3}-\bar{A}_{3} \\
A_{4}-\bar{A}_{4} \\
L-\bar{L}
\end{array}\right]} \\
& \times\left[\begin{array}{ccccc}
225.0492 & -3.7576 & -4.3957 & -2.6474 & 0 \\
-3.7576 & 2.8687 & -0.4963 & -0.2989 & 0 \\
-4.3957 & -0.4963 & 3.9257 & -0.3497 & 0 \\
-2.6474 & -0.2989 & -0.3497 & 1.4240 & 0 \\
0 & 0 & 0 & 0 & 0.4444
\end{array}\right] \\
& \times\left[\begin{array}{cccc}
A_{1}-\bar{A}_{1} \\
A_{2}-\bar{A}_{2} \\
A_{3}-\bar{A}_{3} \\
A_{4}-\bar{A}_{4} \\
L-\bar{L}_{1}
\end{array}\right] \leq 10^{6} .
\end{aligned}
$$

In this problem, the ANSYS software is used to solve to the horizontal displacement $d$ of joint 6 . In order to observe the difference between interval model and ellipsoidal model for reliability analysis results, different values of the maximum allowable displacement $d_{m}$ are taken into account. We calculated the structural reliability with interval model and ellipsoidal model by Monte Carlo simulation with 1000 samples, respectively. The reliability analysis results are given in Figure 12.

As shown in Figure 12, with increasing of $d_{m}$, the nonprobabilistic reliability $R_{c}$ also has an increasing trend. From Figure 12, it is noted that interval model is more conservative than results of ellipsoidal model.

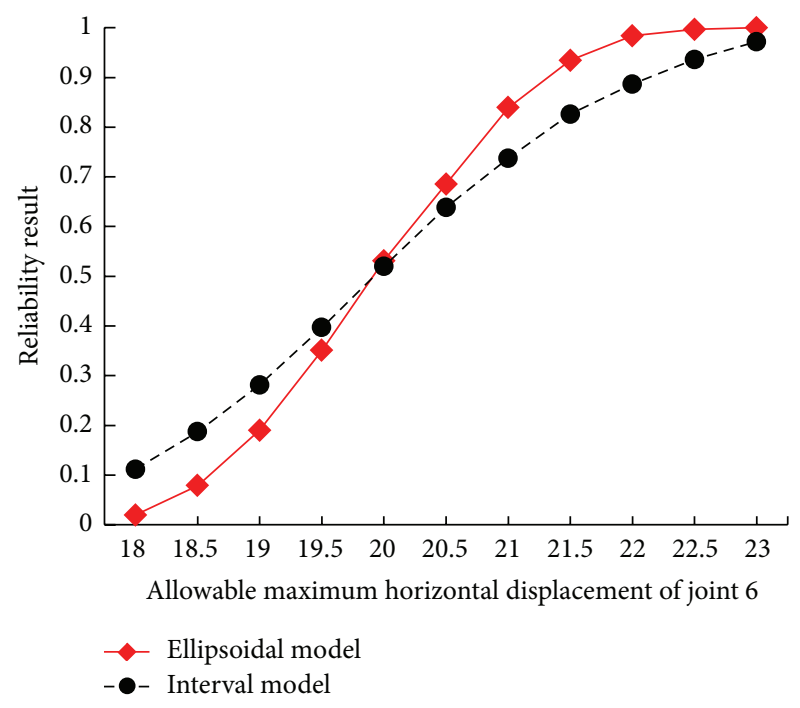

FIGURE 12: Reliability analysis results with interval model and ellipsoidal model.

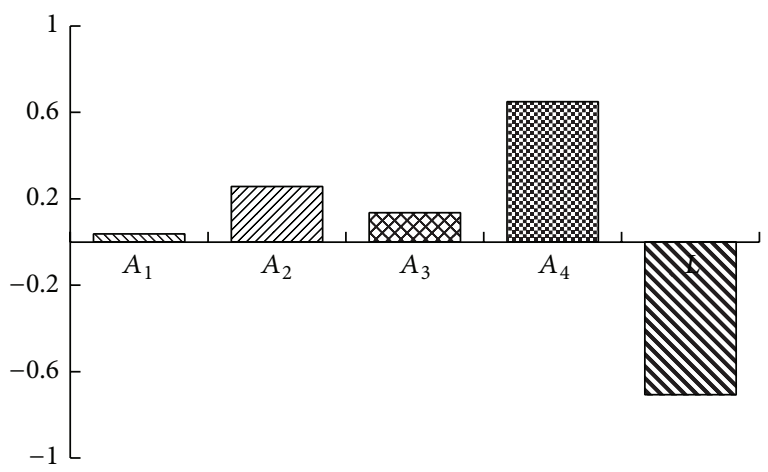

FIgURE 13: Sensitivity analysis result with interval model and ellipsoidal model.

Set the maximum allowable horizontal displacements $d_{m}=22$; perform sensitivity analysis with ellipsoidal model; the structural sensitivity can be obtained readily as shown in Figure 13.

As shown in Figure 13, increasing the value of crosssectional area $A_{i}, i=1,2,3,4$, will enhance the structural rigidity and increase the structural reliability, while increasing the input variable $L$ will decrease the structural reliability. 
TABLE 2: Uncertainty variables of piccolo tube structure.

\begin{tabular}{|c|c|c|c|c|c|}
\hline Variable name & Nominal value & \multirow{2}{*}{\multicolumn{4}{|c|}{$\begin{array}{c}\text { Convex model description } \\
\qquad \delta_{A}^{2} \leq 0.01^{2}\end{array}$}} \\
\hline Pipeline diameter $A(\mathrm{~m})$ & 0.060 & & & & \\
\hline Pipeline wall thickness $B(\mathrm{~m})$ & 0.002 & \multirow{2}{*}{$\delta_{B}, \delta_{C}$} & $\begin{array}{ll}1 & 0\end{array}$ & $\left.\int \delta_{B}\right\}$ & \multirow{2}{*}{$\leq 0.1^{2}$} \\
\hline Hole diameter $C(\mathrm{~m})$ & 0.002 & & 0 & $\delta_{C}$ & \\
\hline Hole side distance $D(\mathrm{~m})$ & 0.040 & \multirow{2}{*}{$\delta_{D}, \delta_{E}$} & $\left.\begin{array}{ll}1 & 0\end{array}\right]$ & $\left\{\delta_{D}\right\}$ & \multirow{2}{*}{$\leq 0.1^{2}$} \\
\hline Hole interval length $E(\mathrm{~m})$ & 0.04 & & 0 & $\delta_{E}$ & \\
\hline Hole angle $F\left(^{\circ}\right)$ & 45 & \multicolumn{4}{|c|}{$\delta_{F}^{2} \leq 0.01^{2}$} \\
\hline Young's modulus $M$ & $2.1 E 11$ & \multicolumn{4}{|c|}{$\delta_{M}^{2} \leq 0.02^{2}$} \\
\hline Density $\rho\left(\mathrm{kg} / \mathrm{m}^{3}\right)$ & 7800 & \multicolumn{4}{|c|}{$\delta_{\rho}^{2} \leq 0.1^{2}$} \\
\hline External vibration frequency $\lambda(\mathrm{Hz})$ & 1700 & \multicolumn{4}{|c|}{$\delta_{\lambda}^{2} \leq 0.1^{2}$} \\
\hline
\end{tabular}

TABLE 3: Anterior four mode frequencies of piccolo tube structure.

\begin{tabular}{lcccc}
\hline Mode number & 1 & 2 & 3 & 4 \\
\hline Natural frequency $(\mathrm{Hz})$ & 1538.4 & 1613.8 & 1819.0 & 1827.5 \\
\hline
\end{tabular}

This sensitivity analysis results are in accordance with the engineering practice.

5.3. Case Study 3. An anti-ice piccolo structure is the core component part of a wing anti-ice system. It is close to aircraft engines, subject to random excitation generated by the engine. So there exists the potential possibility of structural resonances. In this paper, we take a part of anti-ice piccolo structure to perform the resonance reliability and variables sensitivity analysis. The finite element model (FEM) of antiice piccolo structure is shown in Figure 14, which is built with ANSYS software. The local meth model is shown in Figure 15.

The uncertain parameters of piccolo are described with multiellipsoid convex model and are listed in Table 2 .

Firstly, we take the mean value of variables as input parameters. By mode analysis, the anterior four natural frequencies of anti-ice piccolo structure can be obtained. The anterior four mode frequencies are shown in Table 3. The anterior four vibration modes are shown in Figure 16.

As shown in Table 3, the first natural frequency denoted by $\eta$ is close to external excitation frequency. According to the requirement of antiresonance design, the performance function of structural resonance failure can be expressed by

$$
g(\eta, \lambda)=\eta-\lambda
$$

where $\lambda$ is the external excitation frequency and $\eta$ is the first natural frequency. When $|\eta-\lambda| \leq \delta$, the structure will cause resonance damage; when $|\eta-\lambda|>\delta$, structure is safe. In practical engineering, we usually set $\delta=0.05 \lambda$; here $\delta=$ $60 \mathrm{~Hz}$.

To perform Monte Carlo analysis with 365 samples generated by the method mentioned in Section 4.3, the sampling results of performance function are shown in Figure 17.

As shown in Figure 17, there are only 6 samples falling into the structural resonance failure; the pipeline structural reliability can be readily obtained by $R_{c}=350 / 356=$ 0.9863 . To implement the sensitivity analysis by the method

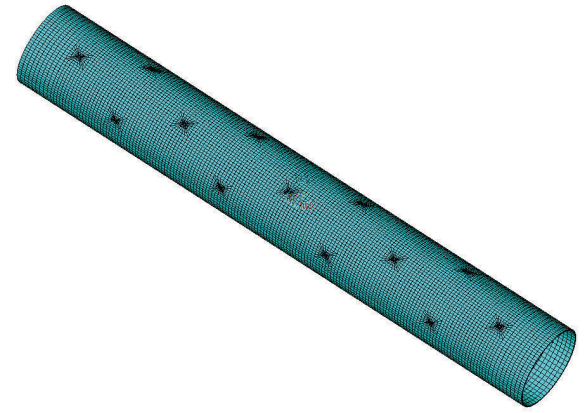

FIGURE 14: FEM of piccolo structure.

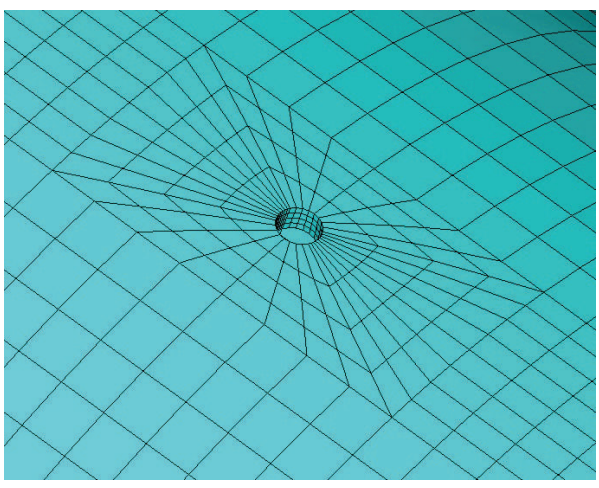

Figure 15: Local meth model of piccolo structure.

mentioned in Section 4.3, the bar chart of sensitivity analysis results is shown in Figure 18.

Figure 18 provides a graphical illustration of the impact of structural input variables on output. A positive sensitivity indicates that increasing the value of input variable will increase the structural reliability. Likewise, a negative sensitivity indicates that increasing the value of input variable will reduce the structural reliability.

\section{Conclusions}

In this paper, a practical nonprobability reliability and global sensitivity analysis method for interval, ellipsoid, and multiellipsoid convex uncertainty model is constructed. In order 


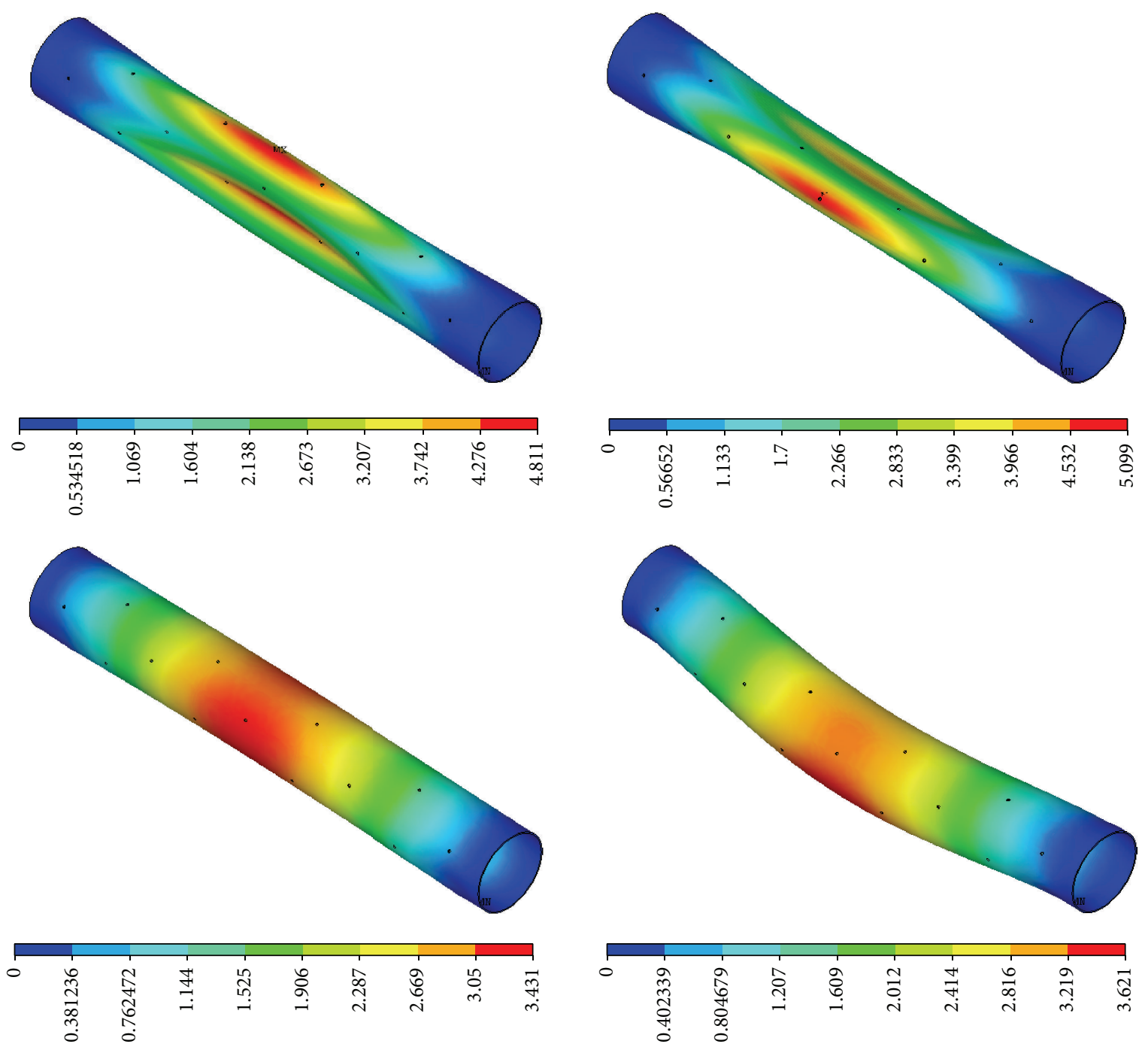

FIGURE 16: The diagram of anterior four vibration modes.

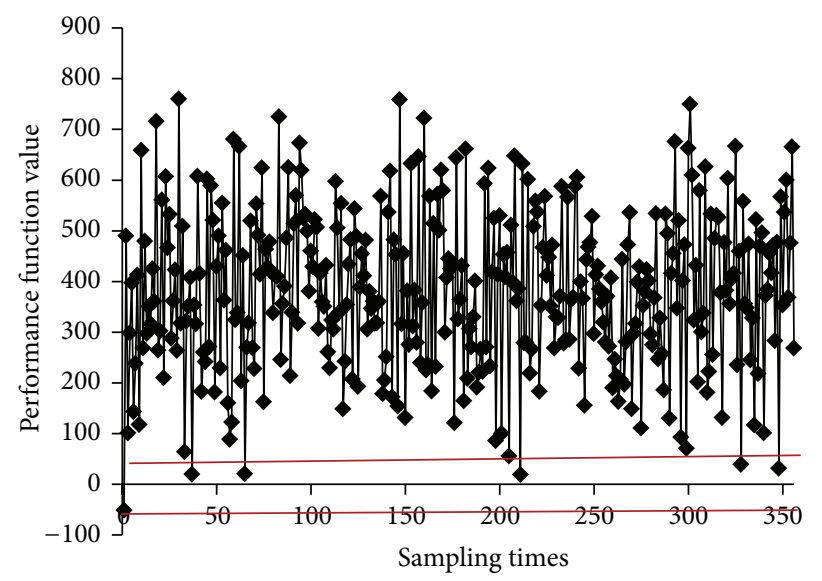

FIGURE 17: Sampling results of performance function. 


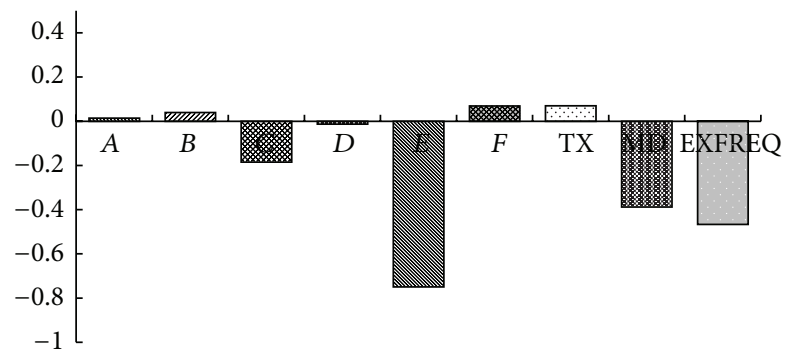

FIGURE 18: Bar chart of sensitivity analysis results.

to provide an accurate depiction of possible model outcome, uniform design, an effective space-filling design, is used to generate representative samples for input variables. As the computation of reliability index proposed in this paper is based on Monte Carlo simulation, it is very suitable for more general engineering problem, like some problems with blackbox performance function.

The global sensitivities analysis method present in this paper is based on Spearman's rank correlation coefficient, which is different from local sensitivities analysis. The advance of this method is that it does not increase the computation cost. After the reliability analysis is implemented, the sensitivity results can be obtained readily. These examples demonstrated the feasibility of the presented method.

\section{Conflict of Interests}

The authors declare that there is no conflict of interests regarding the publication of this paper.

\section{Acknowledgments}

Authors gratefully acknowledge the support of the National Natural Science Foundation of China (E050401) and the Foundation of G2015KY0103.

\section{References}

[1] G. Stefanou, "The stochastic finite element method: past, present and future," Computer Methods in Applied Mechanics and Engineering, vol. 198, no. 9-12, pp. 1031-1051, 2009.

[2] Z. Qiu, D. Yang, and I. Elishakoff, "Probabilistic interval reliability of structural systems," International Journal of Solids and Structures, vol. 45, no. 10, pp. 2850-2860, 2008.

[3] A. D. Kiureghian and O. Ditlevsen, "Aleatory or epistemic? Does it matter?" Structural Safety, vol. 31, no. 2, pp. 105-112, 2009.

[4] A. M. Hasofer and N. C. Lind, "Exact and invariant secondmoment code format," ASCE Journal of the Engineering Mechanics Division, vol. 100, no. 1, pp. 111-121, 1974.

[5] R. Rackwitz and B. Flessler, "Structural reliability under combined random load sequences," Computers and Structures, vol. 9, no. 5, pp. 489-494, 1978.

[6] K. Breitung, "Asymptotic approximations for multinormal integrals," Journal of Engineering Mechanics, vol. 110, no. 3, pp. 357366, 1984.
[7] D. C. Polidori, J. L. Beck, and C. Papadimitriou, "New approximations for reliability integrals," Journal of Engineering Mechanics, vol. 125, no. 4, pp. 466-475, 1999.

[8] R. Y. Rubinstein and D. P. Kroese, Simulation and the MonteCarlo Method, Wiley Series in Probability and Statistics, WileyInterscience, New York, NY, USA, 2nd edition, 2007.

[9] C. Thoft and M. Yoshisuda, Application of Structural Systems Reliability Theory, Springer, Berlin, Germany, 1986.

[10] F. Tonon, "Using random set theory to propagate epistemic uncertainty through a mechanical system," Reliability Engineering \& System Safety, vol. 85, no. 1-3, pp. 169-181, 2004.

[11] C. Jiang, X. Han, and G. R. Liu, "Optimization of structures with uncertain constraints based on convex model and satisfaction degree of interval," Computer Methods in Applied Mechanics and Engineering, vol. 196, no. 49-52, pp. 4791-4800, 2007.

[12] Y. Ben-Haim, "A non-probabilistic concept of reliability," Structural Safety, vol. 14, no. 4, pp. 227-245, 1994.

[13] I. Elishakoff, "Essay on uncertainties in elastic and viscoelastic structures: from A. M. Freudenthal's criticisms to modern convex modeling," Computers and Structures, vol. 56, no. 6, pp. 871-895, 1995.

[14] Y. Ben-Haim and I. Elishakoff, Convex Models of Uncertainties in Applied Mechanics, Elsevier Science, Amsterdam, The Netherlands, 1990.

[15] G. J. Klir, "Generalized information theory: aims, results, and open problems," Reliability Engineering and System Safety, vol. 85, no. 1-3, pp. 21-38, 2004.

[16] C. Jiang, X. Han, G. Y. Lu, J. Liu, Z. Zhang, and Y. C. Bai, "Correlation analysis of non-probabilistic convex model and corresponding structural reliability technique," Computer Methods in Applied Mechanics and Engineering, vol. 200, no. 3336, pp. 2528-2546, 2011.

[17] Y. Ben-Haim and I. Elishakoff, "Discussion on: a nonprobabilistic concept of reliability," Structural Safety, vol. 17, no. 3, pp. 195-199, 1995.

[18] Y. Ben-Haim, "Uncertainty, probability and information-gaps," Reliability Engineering \& System Safety, vol. 85, no. 1-3, pp. 249266, 2004.

[19] Y. Luo, Z. Kang, and Z. Yue, "Maximal stiffness design of twomaterial structures by topology optimization with nonprobabilistic reliability," AIAA Journal, vol. 50, no. 9, pp. 1993-2003, 2012.

[20] S. X. Guo, Z. Y. Lu, and Y. S. Feng, "Non-probabilistic model of structural reliability based on interval analysis," Chinese Journal of Computational Mechanics, vol. 18, no. 1, pp. 56-60, 2001.

[21] J. Tao, C. Jian-Jun, and X. Ya-Lan, "A semi-analytic method for calculating non-probabilistic reliability index based on interval models," Applied Mathematical Modelling, vol. 31, no. 7, pp. 1362-1370, 2007

[22] X. Chen, C.-Y. Tang, C.-P. Tsui, and J. Fan, "Modified scheme based on semi-analytic approach for computing nonprobabilistic reliability index," Acta Mechanica Solida Sinica, vol. 23, no. 2, pp. 115-123, 2010.

[23] Z. Qiu and I. Elishakoff, "Antioptimization of structures with large uncertain-but-non-random parameters via interval analysis," Computer Methods in Applied Mechanics and Engineering, vol. 152, no. 3-4, pp. 361-372, 1998.

[24] I. Elishakoff, R. T. Haftka, and J. Fang, "Structural design under bounded uncertainty-optimization with anti-optimization," Computers and Structures, vol. 53, no. 6, pp. 1401-1405, 1994. 
[25] Z. Kang, Y. Luo, and A. Li, "On non-probabilistic reliabilitybased design optimization of structures with uncertain-butbounded parameters," Structural Safety, vol. 33, no. 3, pp. 196205, 2011.

[26] Y. Luo, A. Li, and Z. Kang, "Reliability-based design optimization of adhesive bonded steel-concrete composite beams with probabilistic and non-probabilistic uncertainties," Engineering Structures, vol. 33, no. 7, pp. 2110-2119, 2011.

[27] Y. Ben-Haim, "A non-probabilistic measure of reliability of linear systems based on expansion of convex models," Structural Safety, vol. 17, no. 2, pp. 91-109, 1995.

[28] C. Jiang, R. G. Bi, G. Y. Lu, and X. Han, "Structural reliability analysis using non-probabilistic convex model," Computer Methods in Applied Mechanics and Engineering, vol. 254, pp. 8398, 2013.

[29] R. Jin, W. Chen, and A. Sudjianto, "An efficient algorithm for constructing optimal design of computer experiments," Journal of Statistical Planning and Inference, vol. 134, no. 1, pp. 268-287, 2005.

[30] R. C. Jin, Enhancements of Metamodeling Techniques in Engineering Design, Graduate College, University of Illinois at Chicago, Chicago, Ill, USA, 2004.

[31] A. Saltelli and J. Marivoet, "Non-parametric statistics in sensitivity analysis for model output: a comparison of selected techniques," Reliability Engineering and System Safety, vol. 28, no. 2, pp. 229-253, 1990.

[32] I. M. Sobol', "Global sensitivity indices for nonlinear mathematical models and their Monte Carlo estimates," Mathematics and Computers in Simulation, vol. 55, no. 1-3, pp. 271-280, 2001.

[33] E. B. Niven and C. V. Deutsch, "Calculating a robust correlation coefficient and quantifying its uncertainty," Computers and Geosciences, vol. 40, pp. 1-9, 2012. 


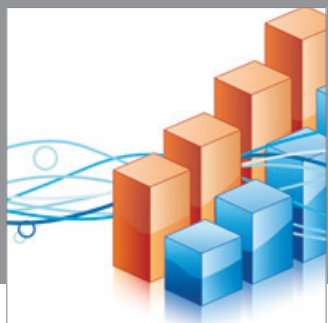

Advances in

Operations Research

mansans

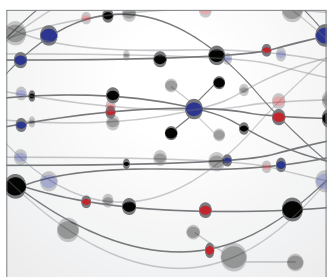

The Scientific World Journal
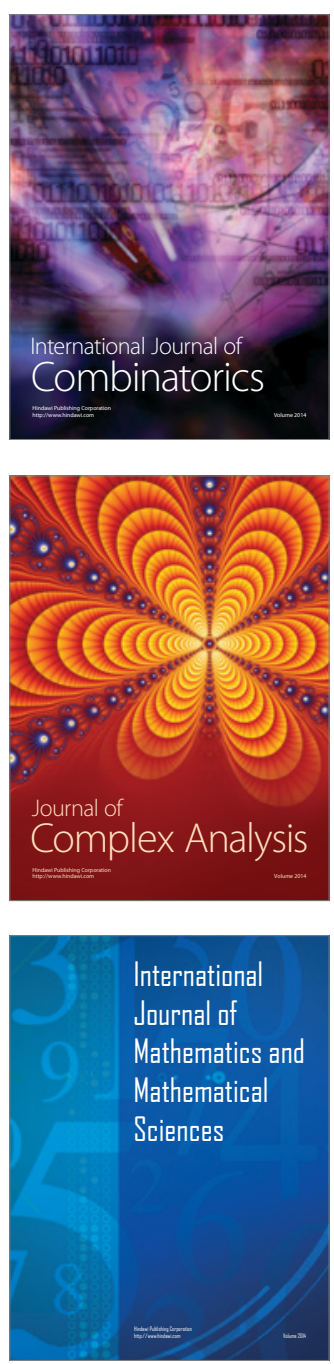
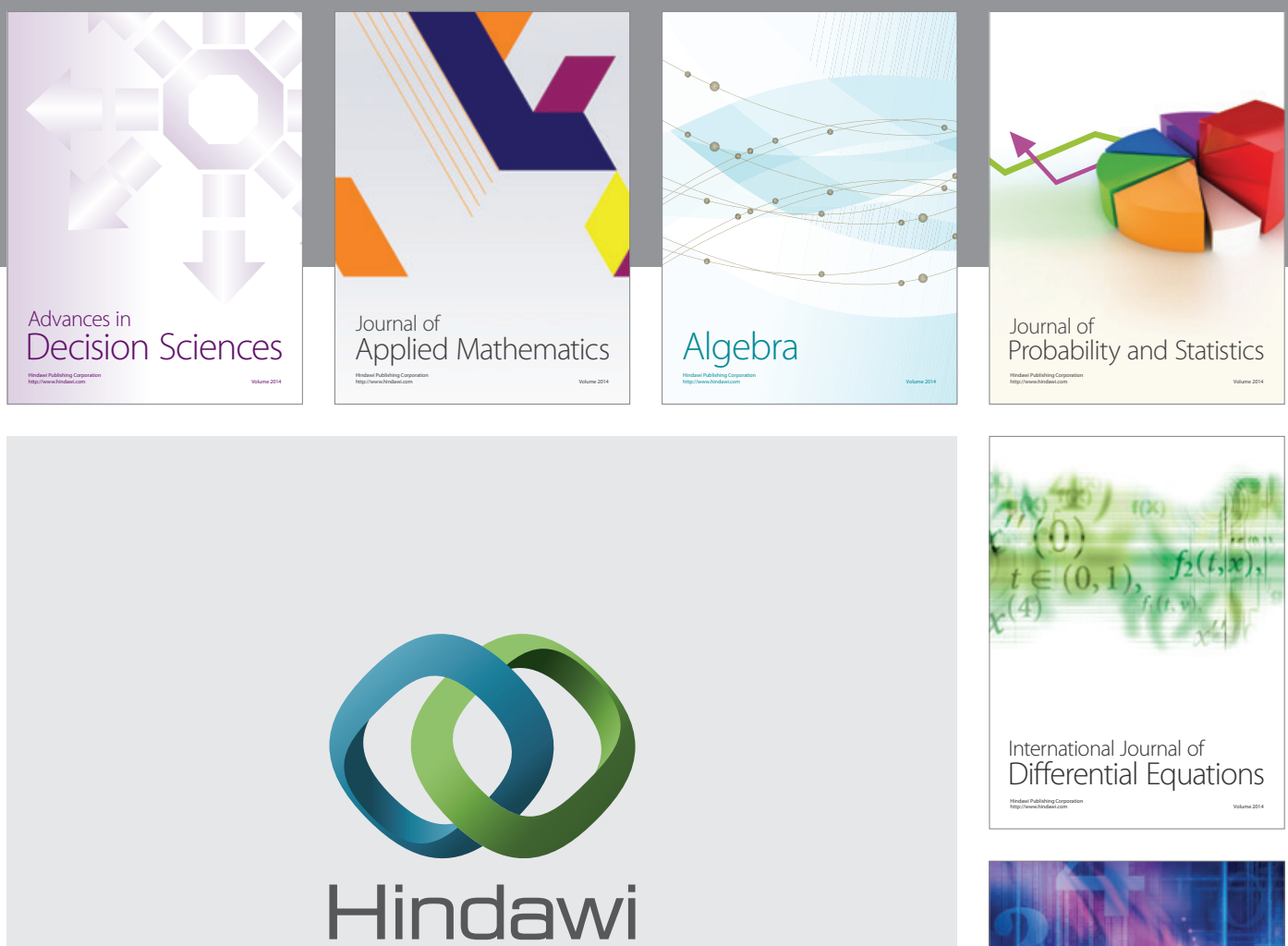

Submit your manuscripts at http://www.hindawi.com
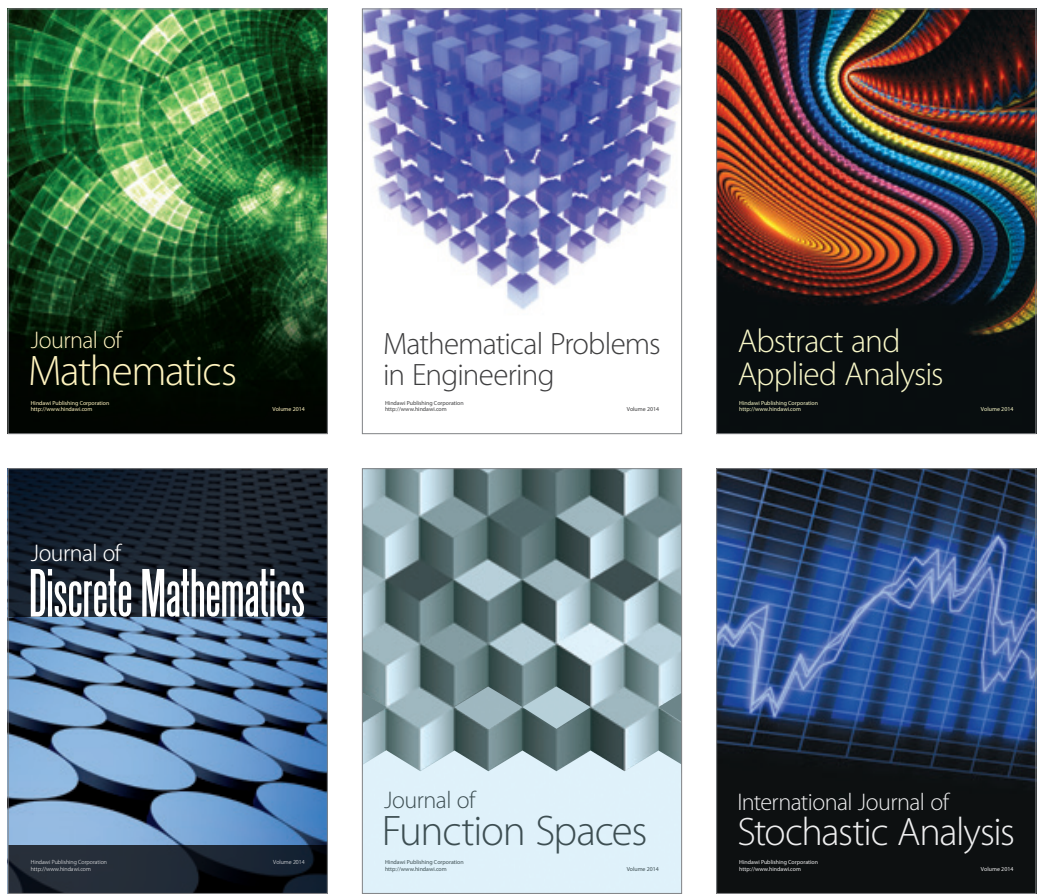

Journal of

Function Spaces

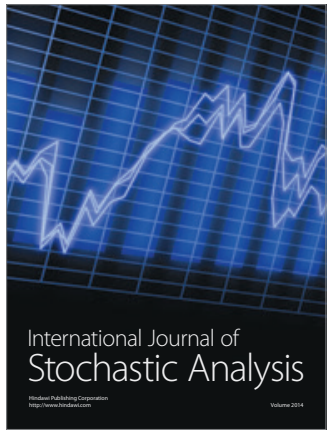

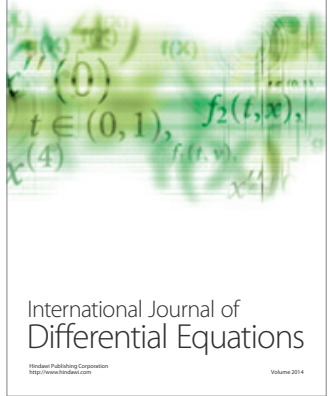
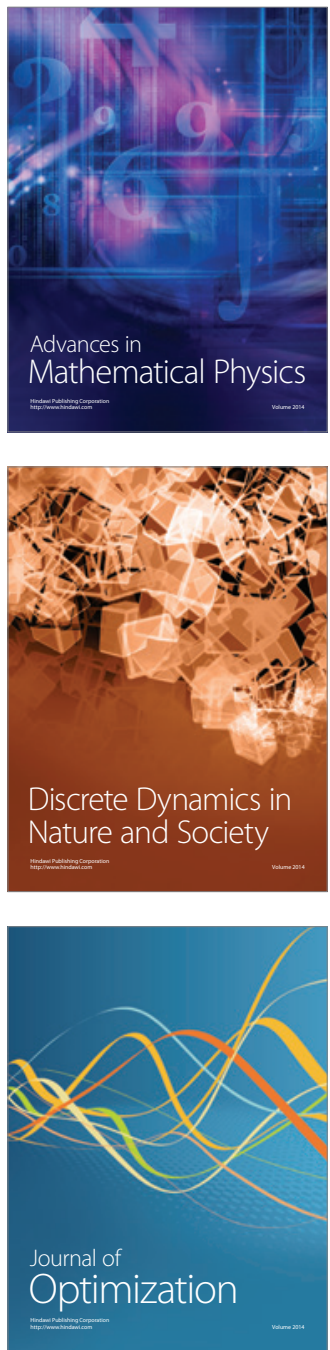